\title{
Does Ownership Make a Difference in Primary Care Practice?
}

\author{
Stephan Lindner, PhD, Leif I. Solberg, MD, William L. Miller, MD, MA, \\ Bijal A. Balasubramanian, MBBS, PhD, Miguel Marino, PhD, \\ K. John McConnell, PhD, Samuel T. Edwards, MD, Kurt C. Stange, MD, PhD, \\ Rachel J. Springer, MS, and Deborah J. Cohen, PhD
}

Purpose: We assessed differences in structural characteristics, quality improvement processes, and cardiovascular preventive care by ownership type among 989 small to medium primary care practices.

Methods: This cross-sectional analysis used electronic health record and survey data collected between September 2015 and April 2017 as part of an evaluation of the EvidenceNOW: Advancing Heart Health in Primary Care Initiative by the Agency for Health Care Research and Quality. We compared physician-owned practices, health system or medical group practices, and Federally Qualified Health Centers (FQHC) by using 15 survey-based practice characteristic measures, 9 survey-based quality improvement process measures, and 4 electronic health record-based cardiovascular disease prevention quality measures, namely, aspirin prescription, blood pressure control, cholesterol management, and smoking cessation support $(\mathrm{ABCS})$.

Results: Physician-owned practices were more likely to be solo $(45.0 \%$ compared with $8.1 \%, P<$ .001 for health system practices and $12.8 \%, P=.009$ for FQHCs) and less likely to have experienced a major change (eg, moved to a new location) in the last year $(43.1 \%$ vs $65.4 \%, P=.01$ and $72.1 \%, P=$ .001 , respectively). FQHCs reported the highest use of quality improvement processes, followed by health system practices. ABCS performance was similar across ownership type, with the exception of smoking cessation support $(51.0 \%$ for physician-owned practices vs $67.3 \%, P=.004$ for health system practices and $69.3 \%, P=.004$ for FQHCs).

Conclusions: Primary care practice ownership was associated with differences in quality improvement process measures, with FQHCs reporting the highest use of such quality-improvement strategies. ABCS were mostly unrelated to ownership, suggesting a complex path between quality improvement strategies and outcomes. (J Am Board Fam Med 2019;32:398-407.)

Keywords: Cardiovascular Diseases, Cross Sectional Analysis, Delivery of Health Care, Group Practice, Ownership, Primary Health Care, Process Measures, Quality Improvement

The organizational structure of primary care practices is rapidly changing in the United States. Primary care can be categorized into 3 major ownership types: independent physician-owned practices, practices employed by hospitals (health system or

This article was externally peer reviewed.

Submitted 13 September 2018; revised 1 February 2019; accepted 5 February 2019.

From Center for Health Systems Effectiveness \& Department of Emergency Medicine, Oregon Health \& Science University, Portland, (SL, KJM); School of Public Health, Oregon Health \& Science University, Portland State University, Portland (SL, MM, KJM); Department of Emergency Medicine, Oregon Health \& Science University, Portland (SL, KJM); HealthPartners Institute, Minneapolis, Minnesota (LIS); Department of Family Medicine, Lehigh medical group practices), and Federally Qualified Health Centers (FQHCs; ie, practices that provide comprehensive primary care to low-income people in underserved communities and that are governed by community boards). Among these, the propor-

Valley Health Network, Allentown, PA (WLM); Department of Epidemiology, Human Genetics, and Environmental Sciences, UTHealth School of Public Health in Dallas, Dallas, TX (BAB); Department of Family Medicine, Oregon Health \& Science University, Portland, (MM STE, RJS, DJC); Section of General Internal Medicine, Veterans Affairs Portland Health Care System, Portland, OR (STE); Center for Community Health Integration, Departments of Family Medicine \& Community Health, Population \& Quantitative Health Sciences, and Sociology, Case Western Reserve University, Cleveland, OH (KCS). 
tion of US physicians employed by hospitals increased from $20 \%$ in 2002 to over $50 \%$ in $2008 .{ }^{1}$ Simultaneously, the number of FQHCs has grown dramatically over the past 20 years in response to higher federal funding, and their role is likely to increase further in the future. ${ }^{2-4}$ At the same time, independent physician-owned practices continue to provide care for millions of Americans. ${ }^{5}$

Despite these changes, we know little about how physician-owned practices, FQHCs, and health system or medical group practices differ in their structural characteristics; approach to quality improvement $(\mathrm{QI})$, including both change management and quality-aligned care delivery processes; and patient outcomes. Health systems and medical groups (ie, practices owned by a hospital and practices owned by nonhospital organizations, respectively) have tended to perform well on process and care quality measures but not across all measures and studies. ${ }^{6-15}$ These studies typically focus on a few measures, 1 or 2 ownership types, and larger practices. Studies on FQHCs have generally been favorable in terms of access, prevention, and quality of care ${ }^{2,16-18}$, but they too typically focus on a few measures and lack explicit comparisons by practice ownership type.

In 2015, the Agency for Health Care Research and Quality (AHRQ) launched EvidenceNOW: Advancing Heart Health in Primary Care. This multiyear, multisite demonstration project tests the effectiveness of external support strategies (eg, practice facilitation) in helping small- to mediumsized primary care practices improve the delivery of preventive care for cardiovascular disease. AHRQ funded 7 regional cooperatives in 12 states that were responsible for recruiting practices and testing various forms of external support. ${ }^{19-21}$ It also funded an independent national evaluation of the overall initiative called Evaluating System Change to Advance Learning and Take Evidence to Scale. ${ }^{22}$ EvidenceNOW Cooperatives recruited 1719 smallto medium-sized primary care practices, defined by AHRQ as practices that provide "integrated, accessible health care services by clinicians who are ac-

Funding: This research was supported by the Agency for Healthcare Research and Quality (AHRQ), grant R01HS023940.

Conflict of interest: none declared.

Corresponding author: Stephan Lindner, PhD, 3030 SW Moody Ave, Portland, OR 97201 (E-mail: lindners@ ohsu.edu). countable for addressing a large majority of personal health care needs". ${ }^{23}$ Recruited practices typically had fewer than 10 clinicians, including physicians, physician assistants, or nurse practitioners. ${ }^{22}$

In this study, we comprehensively compared health system and medical group practices, FQHCs, and physician-owned practices along a rich set of practice characteristics, QI processes, and cardiovascular disease prevention quality outcomes by using a large sample of 923 small- to medium-sized primary care practices that participated in EvidenceNOW. Our goal was to identify differences in these 3 most prevalent primary care ownership types in the United States that might help practice leaders, researchers, and policy makers better understand these practice types to tailor their efforts to improve care where needed.

\section{Methods \\ Data Collection}

This analysis included data collected at baseline (before start of interventions) at each practice. The cooperatives, in collaboration with the initiative Evaluating System Change to Advance Learning and Take Evidence to Scale, developed 2 surveys: a practice survey and a practice member survey. The practice survey assessed practice and patient characteristics (eg, practice size and percent of patients receiving Medicare) and QI processes, including the QI strategy components of the Change Process Capability questionnaire (CPCQ). ${ }^{24}$ In each practice, 1 leader knowledgeable about its structure and approach to QI and care was invited to complete the practice survey. Questions were derived from the National Ambulatory Medical Care Survey (Electronic Medical Records Questionnaire) ${ }^{25}$ and prior primary care research studies. ${ }^{26-31}$ Members of each practice completed the practice member survey.

All members of a practice, which included clinicians, clinical staff (eg, nurses), and administrative staff, were invited to complete the practice member survey. The survey, described elsewhere, ${ }^{32}$ collected information about member characteristics (eg, role and years worked in the practice) and the respondent's perception of how the practice functions. We used 1 measure, a validated single-item burnout question ${ }^{33-35}$ from this survey. The response rate for this measure was $73 \% .^{32}$ Coopera- 
tives were responsible for collecting both practice surveys and practice member surveys from their practices and tailored their collection approach (eg, online or in person) to their local resources and region. Because they recruited practices on a rolling basis, cooperatives collected surveys over a 19month period between September 2015 and April 2017.

Cardiovascular preventive care focused on 4 measures obtained from a practice's electronic health record (EHR) system: aspirin prescription when appropriate, blood pressure control, cholesterol management, and smoking cessation support (the "ABCS"). Participating practices were required to report ABCS data at the practice level quarterly throughout the study period (October 2015 to March 2018). Our baseline ABCS data were collected between October 2015 and March 2017. Before data collection, a collaborative-wide harmonization process specified details of ABCS measurement to ensure consistency across cooperatives.

\section{Study Population}

We selected all practices that (1) submitted both a practice survey and at least 1 practice member survey $(\mathrm{N}=1495$, an $87.0 \%$ response rate); (2) reported being physician-owned, part of a health system or medical group, or having FQHC status $(\mathrm{N}=1236)$; and that (3) had submitted their first ABCS data before intervention start, for a sample size of 989 practices $(57.5 \%$ of 1719 recruited practices). Ownership types not included in this study were academic health center or faculty practices, federal practices (eg, practices belonging to the Veterans Administration), rural health clinics, and Indian health services. We excluded these practices due to the small number of practices with such ownership types in our sample.

\section{Ownership Types}

We defined the 3 ownership types based on a question from the practice survey about the practice's ownership that was adopted from the American Board of Family Medicine's Demographic Survey Questionnaire $^{5,36}$ :

- Health system or medical group practices reported being owned by a hospital, health system, or health maintenance organization;
- FQHC practices reported being FQHCs or look-alikes;

- Physician-owned practices reported being physician-owned practices.

Our analysis focused on differences between these ownership groups. We note that these groupings are just 1 attribute of the practice setting, and within each of these groups, practices differed in terms of structure, resources, function, and patient populations. Most practices named just 1 ownership type but a small number of them $(\mathrm{N}=8)$ reported several. We created mutually exclusive groups that reflected the distinct governance structure of these ownership types. Specifically, we considered all practices owned by a hospital, health system, or health maintenance organization to be health system or medical group practices irrespective of other ownership responses because they were all governed by an outside organization. Of the remaining practices, those with reported FQHC status were considered $\mathrm{FQHC}$ practices whether or not they also indicated being physicianowned because they were all governed by a community-based board of directors.

\section{Measures}

We organized survey variables into practice characteristics, QI process measures, and cardiovascular disease prevention clinical outcome measures. Appendix A provides details for all measures included in the study. Although 989 practices met the study criteria, not all measures had complete information; thus, we reported the number of practices with missing responses by ownership type and each of the individual measures in Appendix A.

\section{Practice Characteristics}

We used 15 practice characteristics categorized as practice demographics, practice patient demographics, and external and internal factors. Practice demographics included whether the practice reported being a solo practice (total practice size was not available because practice size was collected as a categorical variable; see Appendix A), residing in an urban location, being a multispecialty practice, having been under the current ownership for less than 5 years, having experienced at least 1 major change in the last year (eg, new billing system, moved location, and staff turnover), and having less than 5 years of EHR experience. Practice patient 
demographics included the reported percent of patients in a practice receiving Medicaid (which includes Medicaid recipients also eligible for Medicare), the percent of patients receiving Medicare (excluding those also receiving Medicaid), the percent of patients classified as nonwhite and the percent of patients classified as Hispanic or Latino. External and internal factors included whether a practice reported having patient-centered medical home recognition, participating in demonstration programs, such as state innovation model initiatives, being part of an Accountable Care Organization, and having an EHR with stage 1 and 2 meaningful use certification. In addition, the percent of practice members who reported being burned out (defined as the percent of these members reporting a score of 3 or higher on the single-item burnout question from the practice member survey, range 1 to 5) was an internal factor.

\section{QI Process Measures}

Nine QI process measures from the practice survey captured 3 key elements of QI in primary care practices ${ }^{37}$ : quality-aligned care delivery processes, priority, and change management processes. Seven measures covering aspects of quality-aligned care delivery processes included whether the practice reported: (1) that its members routinely discussed clinical quality data, (2) having someone configuring or writing quality reports, (3) producing ABCS clinical quality measures reports in the past 6 months, (4) using at least 1 registry, (5) using empanelment, (6) having implemented cardiovascular disease (CVD) prevention guidelines through standing orders or prompts and reminders, and (7) having implemented CVD management guidelines through standing orders or prompts and reminders. In addition, 1 measure assessed priority (the extent to which a practice's leadership prioritized improving CVD prevention) and 1 measure assessed a practice's ability to manage change (the CPCQ score).

The CPCQ score was based on 14 items assessing practices' use of specific change strategies to improve care (eg, the extent to which a practice delegated tasks from physicians to nonphysicians or empowered those charged with implementing change). It was developed in an iterative modified Delphi process $^{38}$ and has been previously validated. $^{24,39}$ Following a previous study, we calculated the composite CPCQ strategies score as the sum of responses to these 14 items ranging from -2 (strongly disagree) to 2 (strongly agree), resulting in a summary score ranging from -28 to + $28 .^{40}$ The CPCQ score of practices with 1 or more missing response items was set to missing.

\section{Cardiovascular Disease Prevention Clinical Quality Measures}

CVD quality measures included the ABCS-aspirin prescription when appropriate (based on the Center for Medicaid and Medicare's clinical quality measure definition CMS164v4), blood pressure control (CMS165v4), cholesterol management (CMS347v1), and smoking cessation support (CMS138v4). For each of the ABCS measures, practices used EHRs to collect and report the percent of patients receiving treatment among those eligible for treatment.

\section{Study Design and Statistical Analysis}

This investigation was a cross-sectional study examining differences in practice characteristics, QI processes, and cardiovascular disease prevention quality measures by ownership type. We calculated mean or proportions as well as standard errors of these measures to characterize EvidenceNOW practices overall and by ownership groups. We used pairwise $t$ tests to assess statistical significance of differences between health system or medical group and physician-owned practices and between $\mathrm{FQHC}$ and physician-owned practices, respectively. Visual inspection of the distribution of study measures and the large sample size showed the $t$ test to be an appropriate test in this setting. Standard errors were clustered at the cooperative level to account for correlated responses of practices within each of the cooperatives. We used block bootstrap with 1000 iterations because of the small number of clusters. ${ }^{41,42}$ The Institutional Review Board at Oregon Health \& Science University reviewed, approved, and monitored this study, and it is registered as an observational study at clinicaltrials. gov (NCT02560428). We used R version 3.3.1 for our statistical analyses and statistical significance was set at a type I error of $5 \%$.

\section{Results}

More than half of practices in the sample were physician-owned, about one-quarter were owned by health systems or medical groups, and one-fifth reported having FQHC status (Table 1). Physicianowned practices were more likely than the other 2 


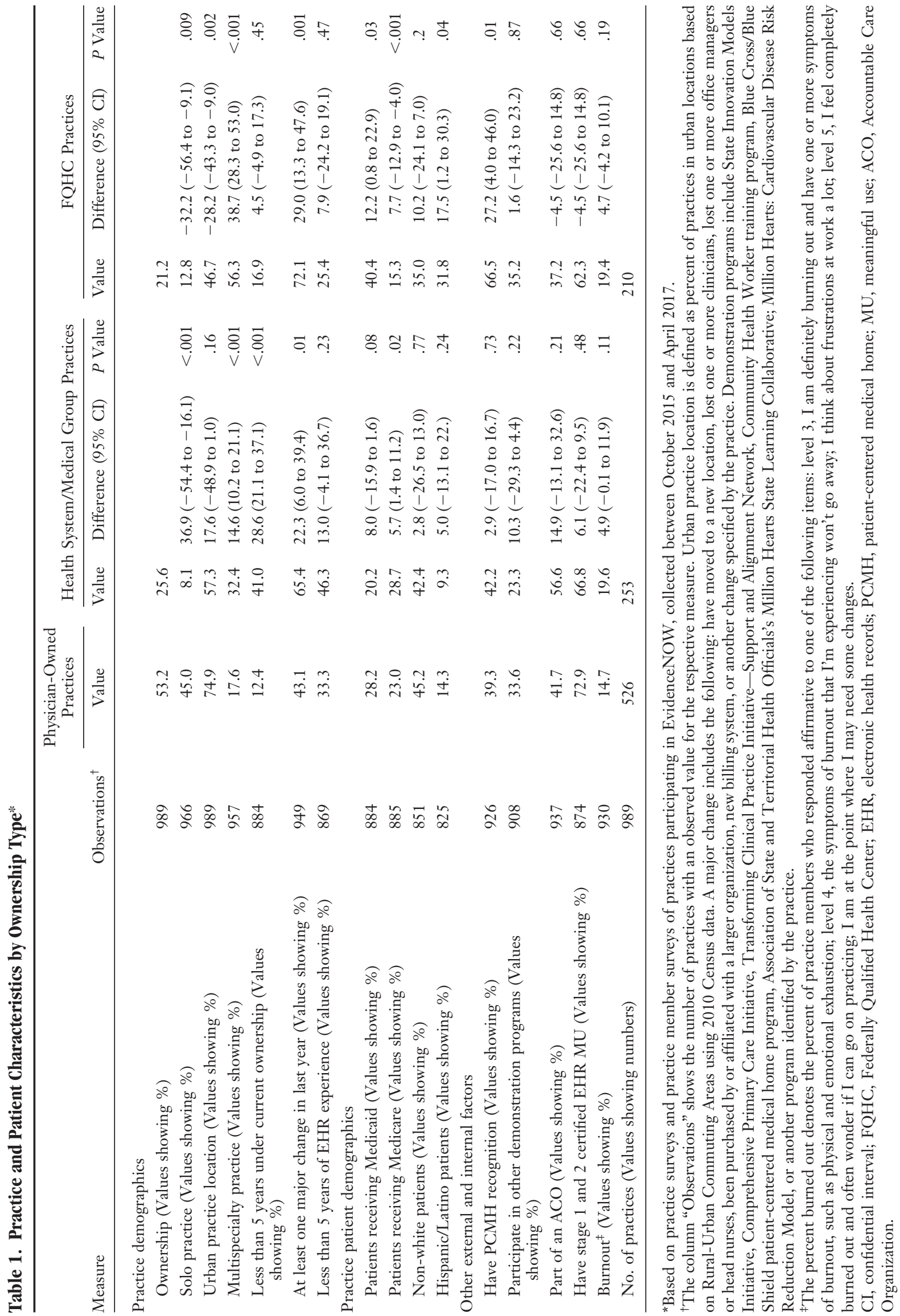


ownership types to report being solo, single-specialty practices located in urban areas. They were also less likely to report having experienced at least 1 major change in the last year. By contrast, health system and medical group practices and FQHCs reported moderately high levels of experiencing a major change in the last year. Health system or medical group practices also had the highest share of Medicare patients. FQHC practices had the lowest share of these patients, the highest share of Medicaid and Hispanic or Latino patients, and by far the highest rate of patient-centered medical home recognition. Burnout was moderately high across all 3 ownership types.

Physician-owned practices less often reported the use of quality-aligned care delivery processes, such as use of registries and CVD care guidelines (Table 2). In contrast, FQHCs stood out in the degree to which they reported using these qualityaligned care delivery processes. Almost all of them reported having someone configure or write quality reports and having produced clinical quality measures reports in the past 6 months. They were also much more likely than the other 2 ownership types to report using at least 1 registry, empanelment, and CVD prevention and management guidelines in EHR prompts or standing orders. Health system practices were intermediate in relation to the use of these of quality-aligned care delivery processes. Differences in the CVD priority measure and CPCQ strategies score were not statistically significant across ownership types.

Regarding CVD quality measures, levels of aspirin prescription when appropriate, blood pressure control, and cholesterol management were similar across ownership types (see Table 3). Health system or medical group practices and FQHCs had higher rates of smoking cessation counseling than physician-owned practices $(51.0 \%$ for physician-owned practices, $67.3 \%, P=.004$ for health system practices and $69.3 \%, P=.004$ for FQHCs).

\section{Discussion}

This large and diverse sample of small- and medium-sized primary care practices provides an important foundation for understanding differences and similarities across the 3 most prevalent primary care ownership models in the United States, that is, physician-owned, health system, and FQHC prac- tices. Ownership groups differed with respect to practice structure and quality-aligned care delivery processes; whereas, the quality of care, as measured by the ABCS, were similar. The only exception in performance was lower rates of smoking cessation counseling among physician-owned practices than FQHC or health system practices, and this result may partly reflect differences in how well smoking counseling is documented across these ownership types. These findings suggest that ownership may be an important factor in understanding how practices engage in QI processes. In what follows, we discuss some possible hypotheses regarding how ownership might have shaped QI processes in our sample of practices.

FQHCs reported the highest use of qualityaligned care delivery processes among all 3 ownership types, which included producing quality reports, using registries, and having EHR prompts or standing orders for CVD prevention and management in place. This finding might reflect practice transformation efforts and reporting requirements by the Health Resources \& Services Administration (HSRA). Other factors that may encourage these level of quality-aligned care delivery processes could include their socioeconomically disadvantaged patient population, participation in learning collaboratives, oversight by a community board, and payment-dependent business model, which includes the need for grant funding and their oversight requirements.

Health system or medical group practices were intermediate in terms of having quality-aligned care delivery processes in place. They did not report a higher change management capacity score than physician-owned practices, suggesting that their management may have provided some QI infrastructure (such as technical personnel to write quality reports) but was less focused on the actual change process in the practice. They were also characterized by a comparatively larger practice size as well as higher level of disruption, which may make it difficult for these practices to implement and sustain quality-aligned care delivery processes. Unlike a recent study conducted by 1 of the cooperatives, we did not find lower levels of burnout among health system practices, ${ }^{43}$ which is likely explained by heterogeneity in the work environment among health system practices across regions, possibly related to how long they have been in the system. 


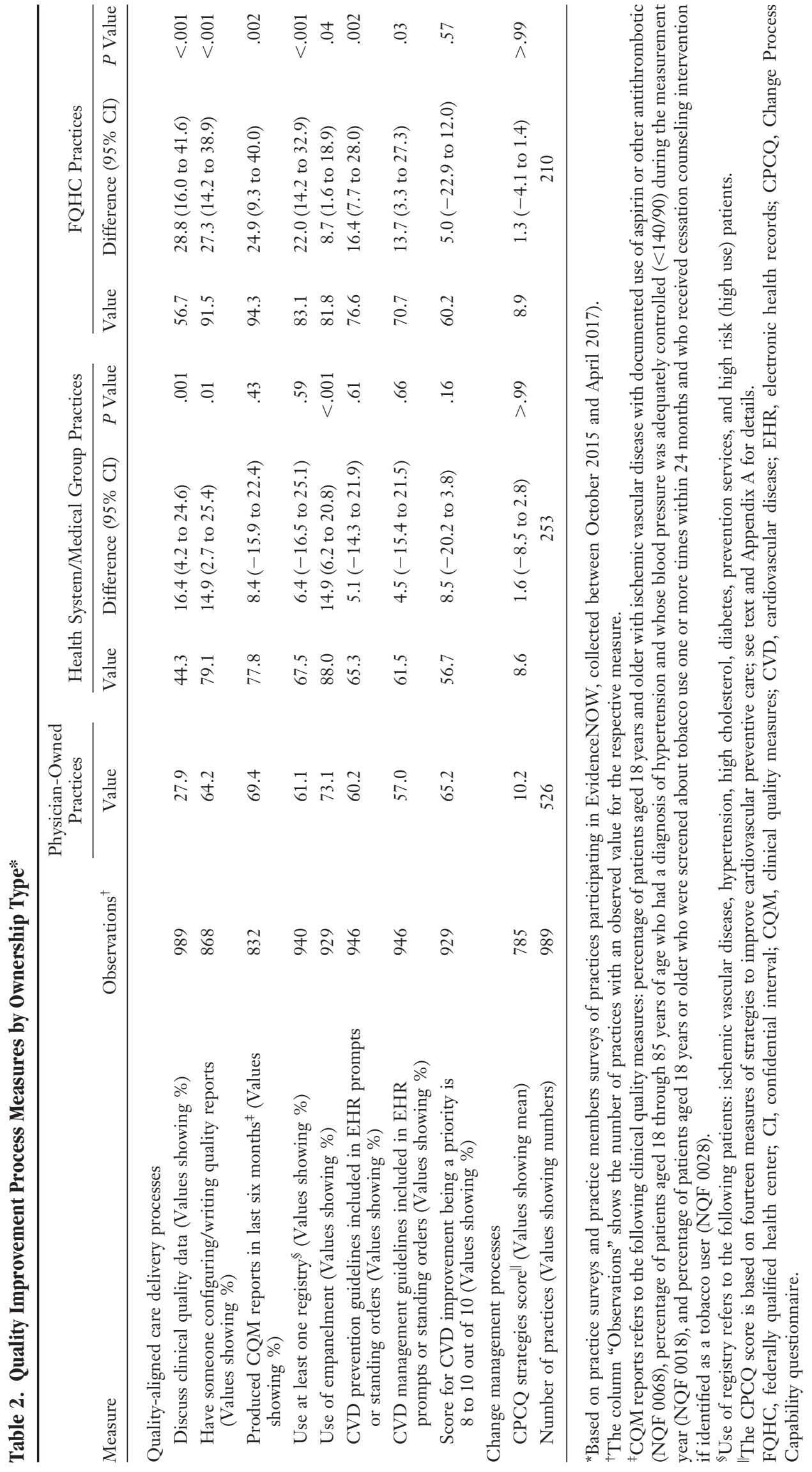


Table 3. Cardiovascular Disease Prevention Clinical Quality Measures by Ownership Type*

\begin{tabular}{|c|c|c|c|c|c|c|c|c|}
\hline \multirow[b]{2}{*}{ Measure } & \multirow{2}{*}{ Observations $^{\dagger}$} & \multirow{2}{*}{$\begin{array}{c}\begin{array}{c}\text { Physician-Owned } \\
\text { Practices }\end{array} \\
\text { Value }\end{array}$} & \multicolumn{3}{|c|}{$\begin{array}{c}\text { Health System/Medical Group } \\
\text { Practices }\end{array}$} & \multicolumn{3}{|c|}{ FQHC Practices } \\
\hline & & & Value & Difference $(95 \% \mathrm{CI})$ & $\begin{array}{c}P \\
\text { value }\end{array}$ & Value & Difference $(95 \% \mathrm{CI})$ & $\begin{array}{c}P \\
\text { value }\end{array}$ \\
\hline $\begin{array}{l}\text { Aspirin (Values } \\
\text { showing \%) }\end{array}$ & 886 & 57.5 & 67.5 & $10.0(0.2$ to 21.5$)$ & .08 & 59.1 & $1.6(-8.2$ to 10.5$)$ & .73 \\
\hline $\begin{array}{l}\text { Blood pressure (Values } \\
\text { showing \%) }\end{array}$ & 892 & 64.0 & 60.6 & $3.4(-7.3$ to 2.0$)$ & .15 & 64.3 & $0.3(-6.4$ to 9.2$)$ & .94 \\
\hline $\begin{array}{l}\text { Cholesterol (Values } \\
\text { showing \%) }\end{array}$ & 679 & 58.2 & 58.2 & $0.1(-8.7$ to 14.3$)$ & .99 & 55.2 & $3.0(-7.9$ to 4.7$)$ & .38 \\
\hline $\begin{array}{l}\text { Smoking (Values } \\
\text { showing \%) }\end{array}$ & 869 & 51.0 & 67.3 & 16.3 (1.8 to 24.2$)$ & .004 & 69.3 & $18.3(1.1$ to 26.7$)$ & .004 \\
\hline $\begin{array}{l}\text { Number of practices } \\
\text { (Values showing } \\
\text { numbers) }\end{array}$ & 989 & 526 & & 253 & & & 210 & \\
\hline
\end{tabular}

*Based on practice surveys of practices participating in EvidenceNOW, collected between October 2015 and April 2017. See Appendix A for a definition of the four cardiovascular disease prevention clinical quality measures.

†The column "Observations" shows the number of practices with an observed value for the respective measure.

CI, confidential interval; FQHC, federally qualified health center.

Physician-owned practices had the lowest levels of quality-aligned care delivery processes in place but may have less need for them due to their small scale. In addition, physician-owned practices may be more likely to use tacit, informal QI processes than the formal and explicit quality-aligned care delivery processes in place at FQHCs and health system practices. Future research could further explore and clarify the relationship between practice size and adoption of quality-aligned processes among primary-care practices.

Despite these clear differences in quality-aligned care delivery processes, levels of ABCS were similar across ownership types, and several hypotheses could explain this finding. The socioeconomically disadvantaged patient population of FQHCs may have motivated them to adopt many quality-aligned care delivery processes but may also have made it difficult for them to achieve a higher quality of care, resulting in similar levels of ABCS compared with the other 2 ownership types. Health system practices may not have achieved higher levels of ABCS because their comparatively higher level of disruption and larger practice size undermined the effectiveness of their quality-aligned care delivery processes. Also, physician-owned practices may have had less need for such processes to achieve comparable levels of care because of their small size. In the end, different levels of quality-aligned care delivery processes may reflect differences in what is needed to achieve the community standard of quality in different practice ownership contexts that reflect different levels of clinician and practice autonomy.

Although this study sheds light on important similarities and differences among various types of small to medium practices, it also has some limitations. This was a cross-sectional study, which does not allow us to identify changes over time as practices changed their ownership. Likewise, we were unable to distinguish whether the higher level of disruption and burnout experienced by health system or medical group practices were ongoing or whether these were transient effects due to an ownership change that dissipated over time. Furthermore, we did not have ABCS for all practices that submitted the practice and practice member survey. However, results for practice structure qualityaligned care delivery processes were similar among the 1222 practices with submitted surveys. Our analysis focused on variation across and not also within these ownership groups and, therefore, does not capture how practices within each ownership type vary in terms of structure, resources, function, and patient populations. Finally, although the numbers of practices were unusually large, participation in EvidenceNOW was voluntary, and our results, therefore, may not necessarily be representative of practices in each region.

We hope that the results of this study and these hypotheses will help provide material for existing studies and proposals but that they will also spur a 
variety of future research projects. For example, researchers could use mixed or qualitative methods to better understand how practices' ownership and associated characteristics shape their care delivery processes and approach to transformation. Such research could, for instance, explore motivating factors behind FQHCs' high use of quality-aligned care delivery processes or describe the effect of disruptions for care delivery processes and care quality. Other future research could investigate differences in practice performance within an ownership category. Although our study highlights substantial differences across ownership types, differences of practices within the same ownership group are also likely to play an important role in the delivery of care.

We are grateful to the participating practices and collaboratives and to Tom Kottke for his valuable insights.

To see this article online, please go to: http://jabfm.org/content/ 32/3/398. full.

\section{References}

1. Kocher R, Sahni NR. Hospitals' race to employ physicians - the logic behind a money-losing proposition. N Engl J Med 2011;364:1790-3.

2. Goldman LE, Chu PW, Tran H, Romano MJ, Stafford RS. Community health centers and private practice performance on ambulatory care measures. Am J Prev Med 2012;43:142-9.

3. Nath JB, Costigan S, Hsia RY. Changes in demographics of patients seen at federally qualified health centers, 2005-2014. JAMA Intern Med 2016;176: 712.

4. Shin P, Sharac J, Barber Z, Rosenbaum SJ, Paradise J. Community health centers: a 2013 profile and prospects as ACA implementation proceeds. Menlo Park, CA: The Henry J. Kaiser Family Foundation, 2015.

5. Liaw WR, Jetty A, Petterson SM, Peterson LE, Bazemore AW. Solo and small practices: a vital. diverse part of primary care. Ann Fam Med 2016;14: $8-15$.

6. Baker LC, Bundorf MK, Kessler DP. Vertical integration: hospital ownership of physician practices is associated with higher prices and spending. Health Aff 2014;33:756-63.

7. Bishop TF, Shortell SM, Ramsay PP, Copeland KR, Casalino LP. Trends in hospital-ownership of physician practices and the effect on processes to improve quality. Am J Manag Care 2016;22:172.

8. Carlin CS, Dowd B, Feldman R. Changes in quality of health care delivery after vertical integration. Health Serv Res 2014;50:1043-1068.
9. Friedberg MW, Coltin KL, Pearson SD, et al. Does affiliation of physician groups with one another produce higher quality primary care? J Gen Intern Med 2007;22:1385-92.

10. Mafi JN, Wee CC, Davis RB, Landon BE. Association of primary care practice location and ownership with the provision of low-value care in the United States. JAMA Intern Med 2017;177:838.

11. Mehrotra A, Epstein AM, Rosenthal MB. Do integrated medical groups provide higher-quality med care than individual practice associations? Ann Intern Med 2006;145:826.

12. Rittenhouse DR, Robin R, Gillies SM, et al. Improving chronic illness care: findings from a national study of care management processes in large physician practices. Med Care Res Rev 2010;67:301-320.

13. Scott KW, Orav EJohn, Cutler DM, Jha AK. Changes in hospital-physician affiliations in U.S. hospitals and their effect on quality of care. Ann Intern Med 2016;166:1-8.

14. Shortell SM, Schmittdiel J, Wang MC, et al. An empirical assessment of high-performing medical groups: results from a national study. Med Care Res Rev 2005;62:407-34.

15. Weeks WB, Gottlieb DJ, Nyweide DJ, et al. Higher health care quality and bigger savings found at large multispecialty medical groups. Health Aff 2010;29: 991-7.

16. Carlson BL, Eden J, O'Connor D, Regan J. Primary care of patients without insurance by community health centers. J Ambul Care Manage 2001;24: 47-59.

17. Politzer RM, Yoon J, Shi L, Hughes RG, Regan J, Gaston MH. Inequality in America: the contribution of health centers in reducing and eliminating disparities in access to care. Med Care Res Rev 2001;58: 234-48.

18. Shi L, Stevens GD. The role of community health centers in delivering primary care to the underserved. J Ambul Care Manage 2007;30:159-70.

19. Parchman ML, Fagnan LJ, Dorr DA, et al. Study protocol for "Healthy Hearts Northwest": a $2 \times 2$ randomized factorial trial to build quality improvement capacity in primary care. Implement Sci 2016; 11:138.

20. Shelley DR, Ogedegbe G, Anane S, et al. Testing the use of practice facilitation in a cluster randomized stepped-wedge design trial to improve adherence to cardiovascular disease prevention guidelines: Healthyhearts NYC. Implement Sci 2015;11:88.

21. Weiner BJ, Pignone MP, DuBard C, et al. Advancing heart health in North Carolina primary care: the Heart Health NOW study protocol. Implement Sci 2015;10:160.

22. Cohen DJ, Balasubramanian BA, Gordon L, et al. A national evaluation of a dissemination and implementation initiative to enhance primary care practice capacity and improve cardiovascular disease care: the 
ESCALATES study protocol. Implement Sci 2015; 11:86.

23. Agency for Healthcare Research and Quality. AHRQ centers for primary care practice-based research and learning. Available from: https://www. ahrq.gov/professionals/systems/primary-care/ rescenters/index.html. Published 2012. Accessed January 30, 2019.

24. Solberg LI, Asche SE, Margolis KL, Whitebird RR. Measuring an organization's ability to manage change: the change process capability questionnaire and its use for improving depression care. Am J Med Qual 2008;23:193-200.

25. Hing E Palso K. National ambulatory med care survey: 2013 State and National Summary Tables. Washington, DC: US Department of Health and Human Services; 2013.

26. Balasubramanian BA, Cohen DJ, Clark EC, et al. Practice-level approaches for behavioral counseling and patient health behaviors. Am J Prev Med 2008; 35:S407-S413.

27. Cohen DJ, Balasubramanian BA, Isaacson NF, Clark EC, Etz RS, Crabtree BF. Coordination of health behavior counseling in primary care. Ann Fam Med 2011;9:406-15.

28. Ferrante J, Balasubramanian BA, Hudson SV, Crabtree BF. Principles of the patient-centered medical home and preventive services delivery. Ann Fam Med 2010;8:108-16.

29. Hung DY, Glasgow RE, Dickinson LM, et al. The chronic care model and relationships to patient health status and health-related quality of life. Am J Prev Med 2008;35:S398-S406.

30. Jaen CR, Crabtree BF, Palmer RF, et al. Methods for evaluating practice change toward a patient-centered medical home. Ann Fam Med 2010;8:S9-S20.

31. Balasubramanian BA, Cohen DJ, Davis MM, et al. Learning evaluation: blending quality improvement and implementation research methods to study healthcare innovations. Implement Sci 2015;10:3 1.

32. Edwards ST, Marino M, Balasubramanian BA, et al. Burnout among physicians, advanced practice clini- cians and staff in smaller primary care practices. J Gen Intern Med 2018;33:2138-46.

33. Williams ES, Konrad TR, Linzer M, et al. Physician, practice, and patient characteristics related to primary care physician physical and mental health: results from the physician worklife study. Health Serv Res 2002;37:119-41.

34. Dolan ED, Mohr D, Lempa M, et al. Using a single item to measure burnout in primary care staff: a psychometric evaluation. J Gen Intern Med 2014;30: 582-7.

35. Rohland BM, Kruse GR, Rohrer JE. Validation of a single-item measure of burnout against the Maslach burnout inventory among physicians. Stress and Health 2004;20:75-9.

36. Peterson LE, Blackburn B, Peabody M, O’Neill TR. Family physicians' scope of practice and american board of fam med recertification examination performance. J Am Board Fam Med 2015;28:265-70.

37. Solberg LI. Improving medical practice: a conceptual framework. Ann Fam Med 2007;5:251-6.

38. Solberg LI, Brekke ML, Fazio CJ, et al. Lessons from experienced guideline implementers: attend to many factors and use multiple strategies. Jt Comm J Qual Improv 2000;26:171-88.

39. Solberg LI, Stuck LH, Crain AL, et al. Organizational factors and change strategies associated with medical home transformation. Am J Med Qual 2014; 30:337-44.

40. Balasubramanian BA, Marino M, Cohen DJ, et al. Use of quality improvement strategies among small to medium-size us primary care practices. Ann Fam Med 2018;16:S35-S43.

41. Cameron AC, Gelbach JB, Miller DL. Bootstrapbased improvements for inference with clustered errors. Rev Econ Stat 2008;90:414-27.

42. Cameron AC, Miller DL. A practitioner's guide to cluster-robust inference. J Hum Resour 2015;50: 317-372.

43. Cuellar A, Krist AH, Nichols LM, Kuzel AJ. Effect of practice ownership on work environment, learning culture, psychological safety, and burnout. Ann Fam Med 2018;16:S44-S51. 


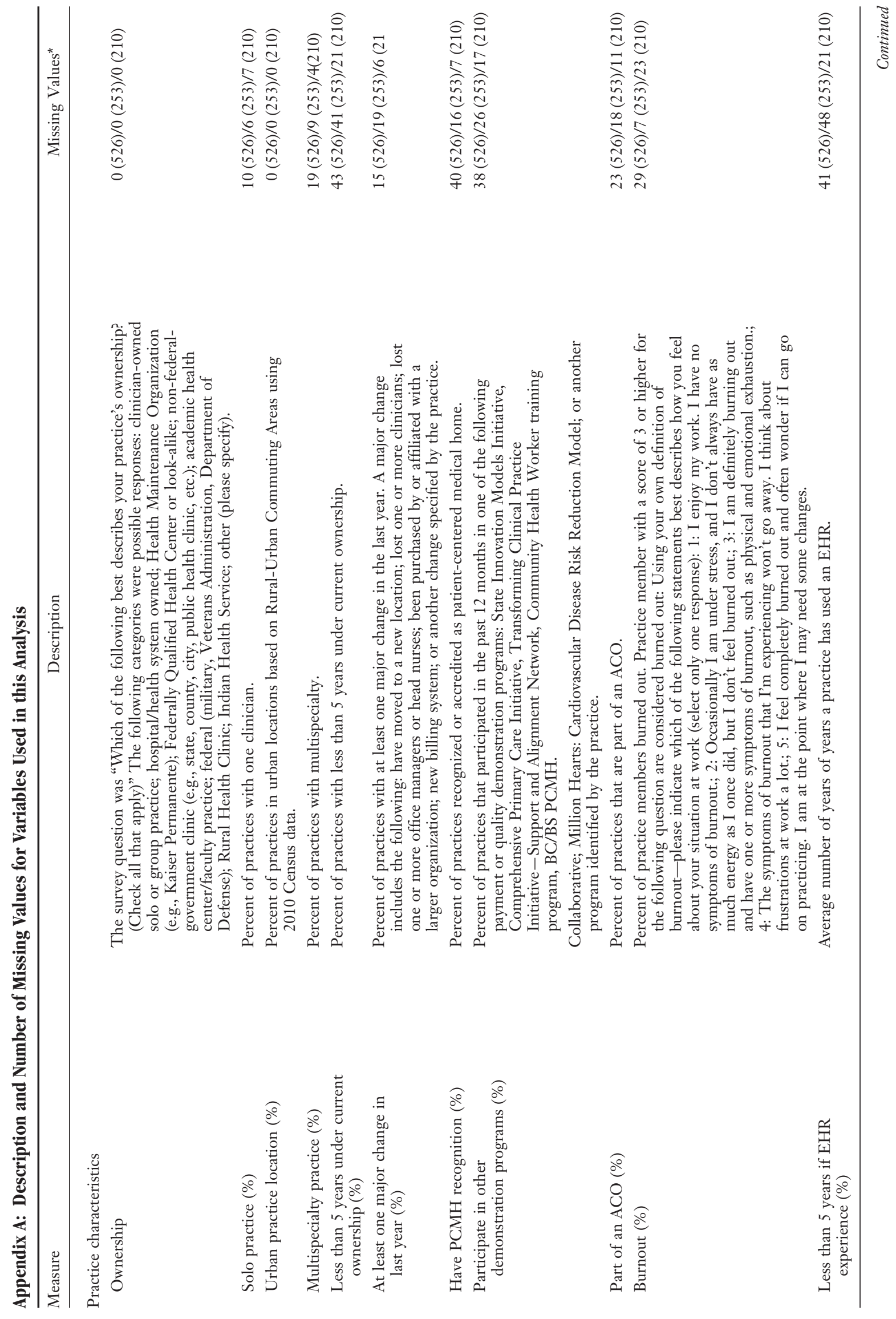




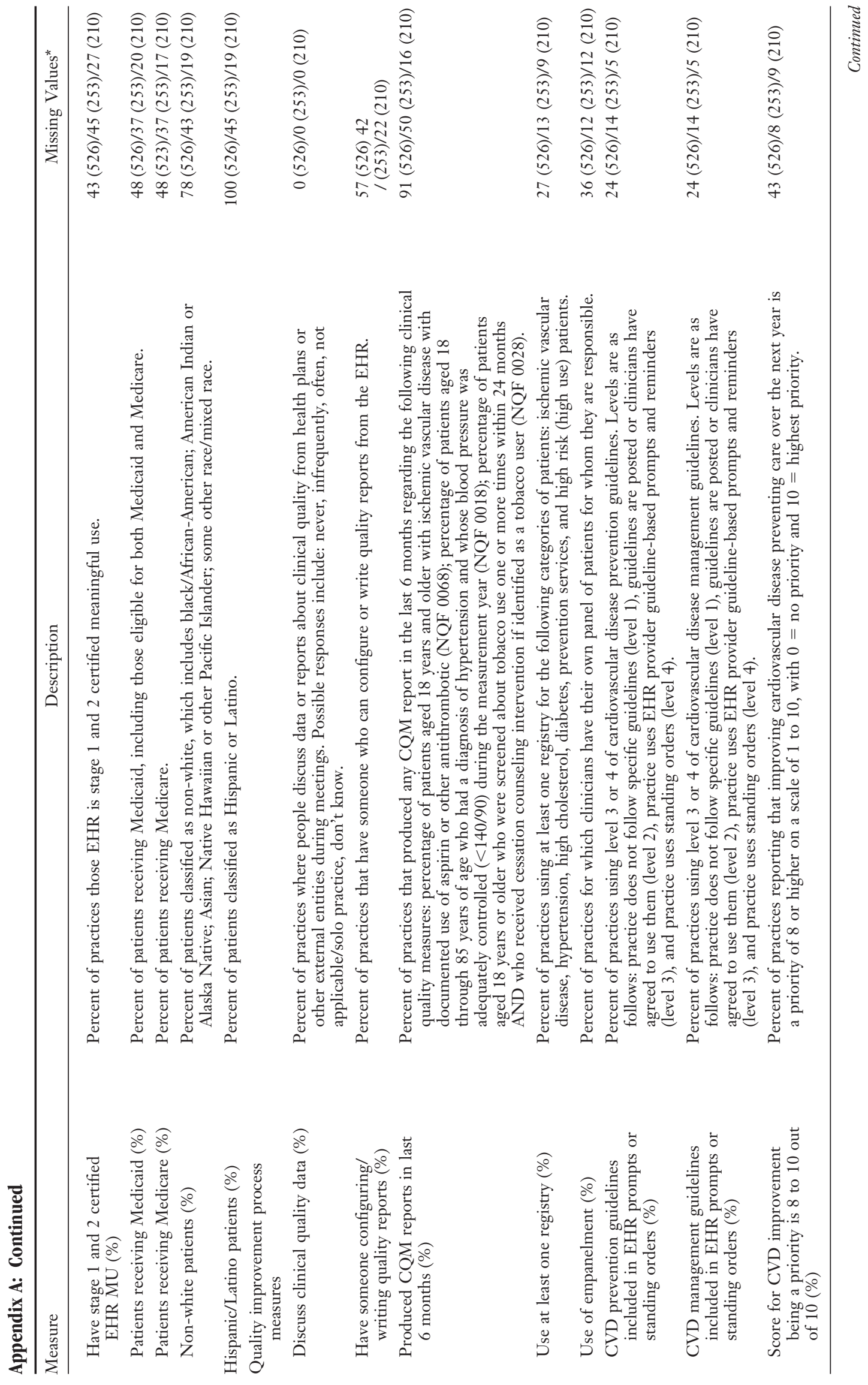

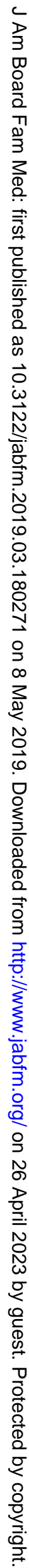




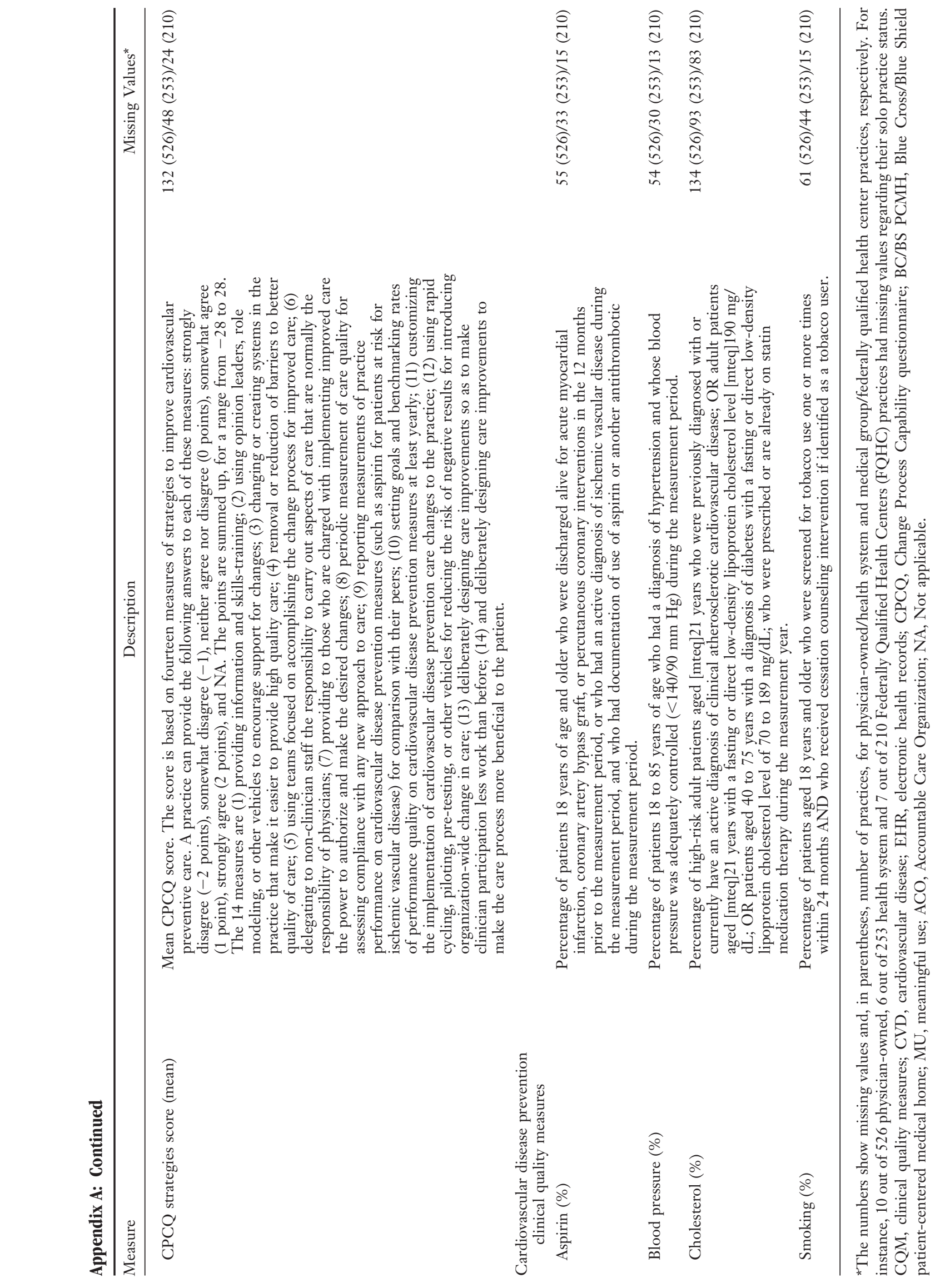

